\title{
Register nominum ad Vol. 149
}

Adler, U., 274 (S) Akerman, R., 71 Amino, E., v. Magara, M. Antoine, T., 206 (B)

Baradlai Bank, E., 26

Bednár, V., und Slunský, R., 369

Bedoya, J. M., Suppl. 148

Berde, B., 207 (B)

Berge, B. S. ten, 40, 112

Berger, J., 279 (S)

Berger, M., 312 (S)

Bertrand, P., et Leulier, J., Suppl. 93

Branger, A., v. Stamm, H.

Brewer, J. I., 79 (B)

Brönnimann, R. und Raaflaub, W.,

312 (S) Brüning, E. J., 144 (B)

Carter, B., v. Jones, C. P. Caspary, J., v. Thiery, M. Catterall, R. D. and Nicol, C. S.,

Luksch, F.

Suppl. 1

V., Salamero, F.

Suppl. 87 Cerva, L., v. Chappaz, G.. Cónill-Serra, V., Salamero, F., und

Esteban, J., 98

Eckerling, B., v. Gans, B.

Erneszt, J., 352

-, v. Zoltán, I.

Esteban, J., v. Cónill-Serra, V.

Fels, H. L., 1

Feo, L. G., Suppl. 101

Fortier, L., Suppl. 158 Friedberg, V., v. Stark, G. Fritz, M. A., v. Lang, W. R. Fuchs, K., and Levy, E., 361 Funck Brentano, P., 383

Gans, B., und Eckerling, B., 33

-, Goldman, J. A. and Ovadia, J. I.,

342 Gianoni, P., v. Giovannini, F., Giovannini, F., et Gianoni, P., 145 Glatthaar, E., 315 (S)

Goldman, J. A., v. Gans, B. Göltner, E., 224

Hauser, A., v. Keller, M.

Hauser, G. A., und Däniken, P. von,

290 (S) Heltai, A., Suppl. 80 Hirvonen, L., Jäykkä, S, Peltonen,T.,

und Rauramo, L., 324 (S) Hochuli, E., v. Stöckli, A. Hopf, M. A., v. Pitteloud, J. J.

Inoki, S., Nakanishi, K., and Nakaba-

yashi, T., Suppl. 48 Jäykkä, S., v. Hirvonen, L. Jones, C. P., Carter, B., and Thomas,

W. L., Suppl. 128 
Kean, B. H., Suppl. 97 Keller, M., und Hauser, A., 337 Koch, W., v. Stark, G. Kostic, P., Suppl. 70 Kotsovsky, D., 207 (B)

390

Register nominum

Kïikal, Z., 162

Kupferberg, A. B., Suppl. 114

Lacomme, M., 327 (B)

Lane, V., 89

Lang, W. R., Fritz, M. A., and Men-duke, H., Suppl. 55

Leeb, H., und Ulm, R., 81

Leulier, J., v. Bertrand, P.

Levy, E., v. Fuchs, K.

Lewin, E., 184, 216

Loula, Z., v. Luksch, F.

Luksch, F., Cerva, L. und Loula, Z., 56

Magara, M., Nakamura, J., Amino, E.,

and Nittono, F., Suppl. 143 Málek, J., v. Silinková-Málková, E. Malz, S., 21

McEwen, D. C, Suppl. 63 Menduke, H., v. Lang, W. R. Moracci, E., 143 (B) Morier-Genoud, J., v. Volet, B.

Nakabayashi, T., v. Inoki, S. Nakamura, J., v. Magara, M. Nakanishi, K., v. Inoki, S. Nilsson, L., and Rybo, G., 193 Nittono, F., v. Magara, M. Nold, B., 265

Onghena, G., v. Thiery, M. Ovadia, J. I., v. Gans, B.

Peltonen, T., v. Hirvonen, L. Pitteloud, J. J., et Hopf, M. A., 209 Pytel, L., 131

Raaflaub, W., 320 (S) -, v. Brönnimann, R. Rauramo, L., 284 (S) -, v. Hirvonen, L. Richter, R. H. H., 270 (S)

Rieben, G., 323 (S) Rigó, J., v. Zoltán, I. Rockenschaub, A., 176 Rosenzweig, E., 329 Rybo, G., v. Nilsson, L.

Sager, C. A., 144 (B) Salamero, F., v. Cónill-Serra, V. Schmid, H. H., 327 (B) Siboulet, A.,

Suppl. 139 Silinková-Málková, E., and Málek, J.,

103 Slunský, R., v. Bednár, V.

Stamm, H., 324 (S)

-, und Branger, A., 323 (S)

Stark, G., Koch, W., und Friedberg, V.,

118 Stöckli, A., und Hochuli, E., 311 (S) Szendi, B., 169, 241 Thiery, M., Onghena, G., Caspary, J.,

et Waefelaer, A., Suppl. 154 Thomas, W. L., v. Jones, C. P.

Tomlin. E. M., v. Youngblood, V. H. Turczyท́ski, T., 376 Ulm, R., v. Leeb, H.

Volet, B., et Morier-Genoud, J., 151

Waefelaer, A., v. Thiery, M.

Waldeyer, A., 207 (B)

Wenner, R., 273 (S)

Wespi, H. J., 307 (S)

Willcox, R. R., Suppl. 122

Williams, J. G\, v. Youngblood, V. H.

Winzeler, H., 320 (S) 
Y. Youngblood

V. H.

Tomlin

E. M. and Williams

J. 0 .

Suppl. 76

Zoltán

I.

Erneszt

J.

und Rigó

J.

250 